\title{
Ensemble-averaged entanglement of two-particle states in Fock space
}

\author{
Jan Naudts* and Tobias Verhulst ${ }^{\dagger}$ \\ Departement Fysica, Universiteit Antwerpen, Groenenborgerlaan 171, 2020 Antwerpen, Belgium
}

(Received 3 April 2007; published 12 June 2007)

\begin{abstract}
Recent results, extending the Schmidt decomposition theorem to wave functions of pairs of identical particles, are reviewed. They are used to give a definition of reduced density operators in the case of two identical particles. Next, a method is discussed to calculate time averaged entanglement. It is applied to a pair of identical electrons in an otherwise empty band, and to a pair of bosons in a quadratic model with infinite range hopping. The effect of degeneracy of the spectrum of the Hamiltonian on the average entanglement is emphasized.
\end{abstract}

DOI: 10.1103/PhysRevA.75.062104

PACS number(s): 03.65.Ud, 05.30.-d

\section{INTRODUCTION}

\section{Schmidt decomposition}

Assume that the wave function $\psi\left(\mathbf{x}_{1}, \mathbf{x}_{2}\right)$ describes two distinguishable particles. Then there exist orthonormal bases of wave functions $\phi_{m}\left(\mathbf{x}_{1}\right)$ and $\chi_{m}\left(\mathbf{x}_{2}\right)$ and coefficients $p_{m}$ $\geq 0$ such that $\psi$ can be written as a single sum

$$
\psi=\sum_{m} \sqrt{p_{m}} \phi_{m} \otimes \chi_{m}
$$

This result is known as the Schmidt decomposition theorem. See, for instance [1], theorem 2.7. The reduced density matrices for each of the particles are then given by

$$
\begin{gathered}
\sigma=\sum_{m} p_{m}\left|\phi_{m}\right\rangle\left\langle\phi_{m}\right|, \\
\tau=\sum_{m} p_{m}\left|\chi_{m}\right\rangle\left\langle\chi_{m}\right| .
\end{gathered}
$$

Indeed, one verifies that for any one-particle operator $A$

$$
\begin{aligned}
\operatorname{Tr} \sigma A=\sum_{m} p_{m}\left\langle\phi_{m}|A| \phi_{m}\right\rangle & =\sum_{m} p_{m}\left\langle\phi_{m} \otimes \chi_{m}|A \otimes \mathbb{I}| \phi_{m} \otimes \chi_{m}\right\rangle \\
& =\langle\psi|A \otimes \mathbb{I}| \psi\rangle
\end{aligned}
$$

and similarly

$$
\operatorname{Tr} \tau A=\langle\psi|\mathbb{I} \otimes A| \psi\rangle .
$$

The knowledge of the coefficients $p_{m}$ suffices to calculate the von Neumann entropies

$$
\mathcal{E}(\psi)=-\operatorname{Tr} \sigma \ln \sigma=-\operatorname{Tr} \tau \ln \tau=-\sum_{m} p_{m} \ln p_{m} .
$$

The latter quantity is a measure for the entanglement of the two particles.

\section{Identical particles}

Recently [2-4], the previous result was generalized to pairs of identical particles, described by a wave function $\psi$ in

\footnotetext{
*Electronic address: jan.naudts@ua.ac.be

†Electronic address: tobias.verhulst@ua.ac.be
}

a Fock space. Let $b^{\dagger}(\phi)$ and $b(\phi)$ be the creation and annihilation operators for a particle with wave function $\phi(\mathbf{x})$. Let $|0\rangle$ denote the vacuum state. Then for each two-particle wave function $\psi$ in a Fock space there exists an orthonormal basis of wave functions $\phi_{m}(\mathbf{x})$ in the one-particle Hilbert space and coefficients $p_{m} \geq 0$ such that

$$
\begin{gathered}
\psi=\frac{1}{\sqrt{2}} \sum_{m} \sqrt{p_{m}} b^{\dagger}\left(\phi_{m}\right) b^{\dagger}\left(\phi_{m}\right)|0\rangle \quad \text { (bosons), } \\
\psi=\sqrt{2} \sum_{m} \sqrt{p_{2 m}} b^{\dagger}\left(\phi_{2 m}\right) b^{\dagger}\left(\phi_{2 m+1}\right)|0\rangle \quad \text { (fermions). }
\end{gathered}
$$

If the dimension of the one-particle Hilbert space is odd then the latter expression does not involve all of the basis vectors $\phi_{m}$.

The physical interpretation of this result, in the case of bosons, is that with probability $p_{m}$ the two particles are both in the same state with wave function $\phi_{m}$. In the fermionic case, one of the particles is in the state $\phi_{2 m}$, the other in the state $\phi_{2 m+1}$. It is then obvious to define reduced density matrices $\sigma$ and $\tau$ by

$$
\sigma=\tau=\sum_{m} p_{m}\left|\phi_{m}\right\rangle\left\langle\phi_{m}\right| \quad \text { (bosons), }
$$

and

$$
\begin{gathered}
\sigma=2 \sum_{m} p_{2 m}\left|\phi_{2 m}\right\rangle\left\langle\phi_{2 m}\right| \\
\tau=2 \sum_{m} p_{2 m+1}\left|\phi_{2 m+1}\right\rangle\left\langle\phi_{2 m+1}\right| \quad \text { (fermions). }
\end{gathered}
$$

By convention, $p_{2 n+1}=p_{2 n}$ in the latter case.

In the fermion case these density matrices are far from unique since for any pair $\phi_{2 m}, \phi_{2 m+1}$ the two basis vectors may be interchanged. Nevertheless, the resulting values of the von Neumann entropies of $\sigma$ and $\tau$ are always the same. Hence, in all cases, the quantity

$$
\mathcal{E}(\psi)=-\sum_{n} p_{n} \ln p_{n}
$$

can be used as a measure of entanglement.

In the next sections we reproduce the proofs of Eqs. (9) and (10) and show that the eigenvalues $p_{n}$ of the reduced 
density matrices can be calculated without actually performing the generalized Schmidt decomposition. In this way the quantification of the entanglement of a pair of identical particles is easier than in the case of distinguishable particles.

\section{Linear entropy}

Even the simplified method to obtain the eigenvalues $p_{n}$ may be too difficult for analytical treatment. For this reason we will make use of the linear entropy instead of the von Neumann entropy (11). It is still a measure of entanglement [5], and is given by

$$
\mathcal{E}_{1}(\psi)=\sum_{n} p_{n}\left(1-p_{n}\right)=1-\sum_{n} p_{n}^{2} .
$$

For similar reasons the von Neumann entropy has been replaced by the linear entropy in other papers as well, for instance in [6-12].

The simplification arises as follows. Let $\rho$ be a density matrix with eigenvalues $p_{n}$. Then it is often feasible to calculate $\rho^{2}$ by matrix multiplication while the calculation of $\rho \ln \rho$ usually requires diagonalization of $\rho$. Also calculating the trace of $\rho^{2}$ is usually a feasible task. The linear entropy $\mathcal{E}_{1}(\psi)$ is then obtained as $1-\operatorname{Tr} \rho^{2}$. Note that the quantity $\operatorname{Tr} \rho^{2}$ is called the purity of the density matrix $\rho$.

\section{Average entanglement}

A final simplification comes from averaging the linear entanglement. In principle, the entanglement of two particles depends on time. Rapid fluctuations of entanglement have been reported to occur in vibrational modes of triatomic molecules [13], and between electrons of Rydberg molecules [14]. They have been studied in theoretical models such as the Dicke model [15], a model of coupled kicked tops [16], the Harper Hamiltonian [17], a dimer model [18], BoseEinstein condensates [19], and random two-qubit interactions $[20,21]$. Hence it is obvious to study the time average of the entanglement. In [22] it is shown how to replace the time average of nonlinear quantities such as the entanglement by ensemble averages. This was applied by the present authors to study the entanglement of distinguishable particles [23].

\section{Overview of the paper}

The next section recalls known results about symmetric and antisymmetric matrices. Proofs are given in the Appendictes. The theorems of [2-4] are reproduced and the calculation of the average entanglement is explained. Section III discusses the entanglement of a pair of identical electrons in an otherwise empty band. Section IV demonstrates the importance of degeneracy of the spectrum of the Hamiltonian for a two-boson model. The paper ends with a discussion in Sec. V, followed by two Appendices.

\section{SCHMIDT DECOMPOSITION IN FOCK SPACE}

Known results on symmetric and antisymmetric matrices

Remember that a matrix $M$ is normal if it commutes with its Hermitian conjugate $M^{\dagger}$. The transpose $M^{T}$ of $M$ has ma- trix elements $\left(M^{T}\right)_{m n}=M_{n m}$. The matrix $M$ is symmetric if $M^{T}=M$; it is antisymmetric if $M^{T}+M=0$. Any matrix with complex entries $M$ can be written as $M=V^{\dagger} D U$ with $D$ diagonal and with $U$ and $V$ unitary. This is the singular value decomposition of $M$. A similar result for symmetric matrices is the following theorem. It is known as Takagi's factorization theorem-see [24], or [3], theorem 3.4. See [25], theorem 5.5.1, for the first claim of the theorem.

Theorem 1. Let there be given a square matrix $M$ with complex entries. Then $M$ is symmetric if and only if it can be written as

$$
M=U^{T} D U,
$$

with $D$ diagonal and $U$ arbitrary. The matrix $U$ can be chosen unitary.

Consider for example the matrix $M$, given by

$$
M=\left(\begin{array}{ll}
i & i \\
i & 1
\end{array}\right) .
$$

It is not normal. Still, there exists a unitary matrix $U$, namely

$$
U=\frac{1}{2}\left(\begin{array}{cc}
1+i & -\sqrt{2} \\
1+i & \sqrt{2}
\end{array}\right),
$$

and a diagonal matrix $D=[1,1]-(1+i)[1,-1] / \sqrt{2}$ such that $M=U^{T} D U$. The method to find $U$ is based on the observation that $U$ diagonalizes $M^{\dagger} M$. Indeed, one has

$$
M^{\dagger} M=U^{\dagger} D^{\dagger} D U \text {. }
$$

This observation is essential for the calculations that follow.

The analogous result for antisymmetric matrices is usually formulated for matrices with real entries only. For matrices with complex entries it follows from lemma 1 of [2]. As noted in [26], the theorem below has been known in physics literature for a long time-see [27,28].

Theorem 2. Let there be given an antisymmetric matrix $M$ with complex entries. Then there exists a unitary matrix $U$ such that $M$ can be written as $M=U^{T} D U$, where $D$ has on each row and each column at most one nonvanishing element.

If $M$ is antisymmetric then also $D=\left(U^{T}\right)^{\dagger} M U^{\dagger}$ is antisymmetric. Hence, if $D$ has at most one nonvanishing element on each row and each column, then it can be brought into blockdiagonal form with blocks of size at most two, simply by swapping the order of rows and of columns. That is, $D$ is a block matrix of the form

$$
D=\left[Z_{1}, Z_{2}, \ldots, Z_{x}, 0,0, \ldots\right],
$$

with $Z_{j}$ of the form

$$
Z_{j}=\left(\begin{array}{cc}
0 & z_{1} \\
-z_{1} & 0
\end{array}\right) .
$$

\section{Application to wave functions in Fock space}

Take an arbitrary orthonormal basis of wave functions $\omega_{n}(\mathbf{x})$ in a finite dimensional one-particle Hilbert space. Any two-particle wave function $\psi$ can be written as 


$$
\psi=\sum_{m n} \lambda_{m n} \omega_{m} \otimes \omega_{n}
$$

The matrix of coefficients $\lambda_{m n}$ is denoted $\Lambda$. In the boson case $\Lambda$ is symmetric; in the fermion case it is antisymmetric. Hence, by the previous theorems there exists a unitary matrix $U$ and a matrix $D$, with at most one nonvanishing element on each row and each column, such that $\Lambda=U^{T} D U$. Then one can write

$$
\psi=\sum_{m n r s} U_{r m} D_{r s} U_{s n} \omega_{m} \otimes \omega_{n}=\sum_{r s} D_{r s} \phi_{r} \otimes \phi_{s}
$$

with

$$
\phi_{r}=\sum_{m} U_{r m} \omega_{m}
$$

Because the matrix $D$ has at most one nonvanishing element on each row and each column, the double sum in Eq. (20) reduces to a single sum. This yields Eqs. (7)-(10).

Next observe that

$$
\Lambda^{\dagger} \Lambda=\left(U^{T} D U\right)^{\dagger} U^{T} D U=U^{\dagger} D^{\dagger} D U
$$

Hence the matrices $\Lambda^{\dagger} \Lambda$ and $D^{\dagger} D$ have the same eigenvalues. But the eigenvalues of $D^{\dagger} D$ are precisely the coefficients $p_{n}$ appearing in the expression (11) for the entanglement. Hence, in order to calculate the entanglement of two identical systems, it suffices to expand the wave function $\psi$ in an arbitrary basis, as done in Eq. (19). Next, the matrix of expansion coefficients $\Lambda$ is used to form $\Lambda^{\dagger} \Lambda$. Finally, the eigenvalues $p_{n}$ of the latter matrix are calculated.

\section{Average entanglement using the linear entropy functional}

If now the linear entropy is used to quantify the entanglement instead of the von Neumann entropy then one finds

$$
\mathcal{E}_{1}(\psi)=1-\operatorname{Tr}\left(\Lambda^{\dagger} \Lambda\right)^{2}
$$

Next assume that the basis of eigenvectors $\psi_{n}$ diagonalizes the Hamiltonian $H$. One can expand an arbitrary wave function $\psi$ in this basis

$$
\psi=\sum_{n} \sqrt{p_{n}} e^{i \chi_{n}} \psi_{n}
$$

with real phases $\chi_{n}$ and positive coefficients $p_{n}$ satisfying $\Sigma_{n} p_{n}=1$. With each basis vector $\psi_{n}$ corresponds an antisymmetric matrix $\Lambda^{(n)}$ via Eq. (19). One then obtains

$$
\begin{aligned}
\mathcal{E}_{1}(\psi)= & 1-\sum_{m n r s} \sqrt{p_{m} p_{n} p_{r} p_{s}} e^{i\left(\chi_{n}-\chi_{m}\right)} e^{i\left(\chi_{s}-\chi_{r}\right)} \\
& \times \operatorname{Tr} \Lambda^{(m)^{\dagger}} \Lambda^{(n)} \Lambda^{(r)^{\dagger}} \Lambda^{(s)} .
\end{aligned}
$$

Assume now that the spectrum of $H$ is nondegenerate. Then the time-average entanglement of $\psi$ may be calculated as an ensemble average, by integrating over the phase factors in the above expression. The result is

$$
\begin{aligned}
\overline{\mathcal{E}_{1}(\psi)}= & 1-\sum_{m, r} p_{m} p_{r} \operatorname{Tr} \Lambda^{(m)^{\dagger}} \Lambda^{(m)} \Lambda^{(r)^{\dagger}} \Lambda^{(r)} \\
& -\sum_{m, n} p_{m} p_{n} \operatorname{Tr} \Lambda^{(m)^{\dagger}} \Lambda^{(n)} \Lambda^{(n)^{\dagger}} \Lambda^{(m)} \\
& +\sum_{m} p_{m}^{2} \operatorname{Tr}\left(\Lambda^{(m)^{\dagger}} \Lambda^{(m)}\right)^{2}=S_{1}(\sigma)+S_{1}(\tau)-\Delta,
\end{aligned}
$$

with

$$
\sigma=\sum_{m} p_{m} \Lambda^{(m)^{\dagger}} \Lambda^{(m)}
$$

$$
\begin{gathered}
\tau=\sum_{n} p_{n} \Lambda^{(n)} \Lambda^{(n)^{\dagger}}, \\
\Delta=1-\sum_{m} p_{m}^{2} \operatorname{Tr}\left(\Lambda^{(m)^{\dagger}} \Lambda^{(m)}\right)^{2} .
\end{gathered}
$$

Note that $\Lambda^{(m)^{\dagger}} \Lambda^{(m)}$ and $\Lambda^{(m)} \Lambda^{(m)^{\dagger}}$ have the same eigenvalues. Hence one has always $S_{1}(\sigma)=S_{1}(\tau)$.

The entanglement $\overline{\mathcal{E}_{1}(\psi)}$ calculated above depends on the choice of the basis of eigenfunctions of the Hamiltonian. When the spectrum is nondegenerate then these eigenfunctions are unique up to a complex phase factor, which has no influence on the entanglement. Hence the problem of nonuniqueness occurs only when the spectrum is degenerate. In that case the decomposition (24) of $\psi$ into eigenfunctions should be replaced by

$$
\psi=\sum_{n} \sqrt{p_{n}} \psi_{n}^{\prime}
$$

with

$$
\psi_{n}^{\prime}=\frac{F_{n} \psi}{\left\|F_{n} \psi\right\|} \quad \text { and } \quad p_{n}=\left|\left\langle\psi_{n} \mid \psi\right\rangle\right|^{2} .
$$

Here, the $F_{n}$ are the orthogonal projections onto the degenerate eigenspaces of the two-particle Hamiltonian.

Examples of degeneracy are discussed below.

\section{FERMION MODEL}

As a first application of our method we consider the average entanglement of a pair of identical electrons in an otherwise empty conduction band. A suitable description is given by the one-dimensional Hubbard model. There is an extended literature about this model. Its study accelerated after Lieb and $\mathrm{Wu}[29,30]$ showed that its spectrum can be calculated using the Bethe ansatz. For a review paper see [31]. In our treatment here both electrons have the same spin. Hence the Hamiltonian can be simplified to

$$
H=-\sum_{j, k=1}^{N} t_{j k} b_{j}^{\dagger} b_{k},
$$

where $b_{k}$ is the annihilation operator for an electron at site $k$ and the conjugate $b_{k}^{\dagger}$ is the creation operator. The coefficients $t_{j k}$ satisfy 


$$
t_{j, j+1}=t_{j, j-1}=1 \quad \text { and } \quad t_{j, k}=0 \text { otherwise. }
$$

Periodic boundary conditions are assumed, identifying site $N$ with site 0 .

We will show that the average entanglement of the two electrons is a nontrivial conserved quantity of this model.

\section{Entanglement of the eigenvectors}

Consider a wave function $\psi$ describing two identical electrons, say, both with spin up, in an otherwise empty band. Then $\psi$ is an eigenvector of $H$, with eigenvalue $\epsilon$, if and only if the antisymmetric matrix $\Lambda$ of coefficients $\lambda_{m n}$ satisfies the matrix equation

$$
T \Lambda+\Lambda T=-\epsilon \Lambda .
$$

In the one-dimensional model with nearest neighbor interactions [i.e., $\left.t_{m n}=A\left(\delta_{m, n+1}+\delta_{m+1, n}\right)\right]$ and with periodic boundary conditions [i.e., $t_{N-1,0}=t_{0, N-1}=A$ ] the solutions are parametrized with two integers $r$ and $s$, with $r \neq s$, and are given by

$$
\lambda_{m n}^{(r s)}=\frac{1}{N \sqrt{2}}[\theta(m r+n s)-\theta(n r+m s)]
$$

with $\theta(m)=\exp (2 \pi i m / N)$. The corresponding eigenvalue is then

$$
E^{(r s)}=-2 \operatorname{Re} \theta(r)-2 \operatorname{Re} \theta(s) .
$$

Note that $\Lambda^{(r s)}=-\Lambda^{(s r)}$.

With the explicit expression (35) it is straightforward to calculate

$$
\begin{aligned}
{\left[\Lambda^{\left.(r s)^{\dagger} \Lambda^{(r s)}\right]_{m n}=}\right.} & \frac{1}{2 N^{2}} \sum_{t} \overline{[\theta(t r+m s)-\theta(m r+t s)]} \\
& \times[\theta(t r+n s)-\theta(n r+t s)] \\
= & \frac{1}{2 N}[\overline{\theta(m s)} \theta(n s)+\overline{\theta(m r)} \theta(n r)] .
\end{aligned}
$$

Hence, one obtains

$$
\begin{aligned}
\operatorname{Tr}\left[\Lambda^{(r s)^{\dagger}} \Lambda^{(r s)}\right]^{2}= & \frac{1}{4 N^{2}} \sum_{m n}[\overline{\theta(m s)} \theta(n s)+\overline{\theta(m r)} \theta(n r)] \\
& \times[\overline{\theta(n s)} \theta(m s)+\overline{\theta(n r)} \theta(m r)]=\frac{1}{2} .
\end{aligned}
$$

One concludes that all two-particle eigenvectors $\psi^{(r s)}$ are entangled, with $\mathcal{E}_{1}\left(\psi^{(r s)}\right)=1 / 2$.

One can do even more. The vectors $u^{ \pm}$with components

$$
u_{m}^{ \pm}=\overline{\theta(m r)} \pm \overline{\theta(m s)}
$$

are eigenvectors of the matrix $\Lambda^{(r s)^{\dagger}} \Lambda^{(r s)}$ with eigenvalue $1 / 2$. All other eigenvectors have eigenvalue 0 . Hence, with the notations of previous sections, the only nonvanishing eigenvalues are $p_{0}=p_{1}=1 / 2$. The entanglement of the twoparticle eigenvectors $\psi^{(r s)}$, using the von Neumann entropy, is therefore

$$
\mathcal{E}\left(\psi^{(r s)}\right)=2\left(-\frac{1}{2} \ln \frac{1}{2}\right)=\ln 2 .
$$

\section{Average entanglement}

Let us now calculate the average entanglement of an arbitrary two-particle wave function. One has

$$
\Delta=1-\frac{1}{2} \sum_{r s} p_{r s}^{2}
$$

Similarly,

$$
\begin{aligned}
\operatorname{Tr}\left[\Lambda^{(r s)^{\dagger}} \Lambda^{(r s)}\right]\left[\Lambda^{\left(r^{\prime} s^{\prime}\right)^{\dagger}} \Lambda^{\left(r^{\prime} s^{\prime}\right)}\right] \\
=\frac{1}{4 N^{2}} \sum_{m n}[\overline{\theta(m s)} \theta(n s)+\overline{\theta(m r)} \theta(n r)]\left[\overline{\theta\left(n s^{\prime}\right)} \theta\left(m s^{\prime}\right)\right. \\
\left.\quad+\overline{\theta\left(n r^{\prime}\right)} \theta\left(m r^{\prime}\right)\right]=\frac{1}{4}\left[\delta_{s s^{\prime}}+\delta_{r r^{\prime}}+\delta_{r s^{\prime}}+\delta_{s r^{\prime}}\right] .
\end{aligned}
$$

Hence

$$
S(\sigma)=S(\tau)=1-\frac{1}{4} \sum_{r r^{\prime} s s^{\prime}} p_{r s} p_{r^{\prime} s^{\prime}}\left[\delta_{s s^{\prime}}+\delta_{r r^{\prime}}+\delta_{r s^{\prime}}+\delta_{s r^{\prime}}\right] .
$$

Using Eq. (26) and the normalization condition

$$
\sum_{r>s} p_{r s}=1
$$

one calculates

$$
\begin{aligned}
\overline{\mathcal{E}_{1}(\psi)}= & \frac{1}{2}+\frac{1}{2}\left[\sum_{r>s} p_{r s}\right]^{2}+\frac{1}{2} \sum_{r s} p_{r s}^{2}-\frac{1}{2} \sum_{r r^{\prime} s s^{\prime}} p_{r s} p_{r^{\prime} s^{\prime}} \\
& \times\left[\delta_{s s^{\prime}}+\delta_{r r^{\prime}}+\delta_{r s^{\prime}}+\delta_{s r^{\prime}}\right] \\
= & \frac{1}{2}+\sum_{r r^{\prime} s s^{\prime}}^{\prime} p_{r s} p_{r^{\prime} s^{\prime}},
\end{aligned}
$$

where the summation $\Sigma^{\prime}$ is restricted to the sets of indices $r r^{\prime} s s^{\prime}$ satisfying $r>r^{\prime}, s>s^{\prime}, r \neq s, r \neq s^{\prime}, r^{\prime} \neq s$, and $r^{\prime}$ $\neq s^{\prime}$.

In the above calculation the degeneracy of the spectrum has been neglected. As a consequence, the result is only valid when the projection of $\psi$ on any of the degenerate subspaces is always parallel to one of the basis vectors $\psi^{(r s)}$. This is not the case in general. The calculation of the entanglement of an arbitrary wave function is therefore more complicated. We will not treat this general case but end this section with an example where degeneracy does not play a role. The complications due to degeneracy will be discussed in the bosonic example of the next section.

\section{Example with $N=4$}

Take $N=4$. This means that the two electrons occupy four sites on a ring. The eigenvalues are $-2,0,2$, each twofold degenerate. The corresponding eigenvectors are $\psi^{(1,4)}$ and $\psi^{(3,4)}, \psi^{(1,3)}$ and $\psi^{(2,4)}$, and $\psi^{(1,2)}$ and $\psi^{(2,3)}$. We neglect the effect of the degeneracy on the average entanglement with the argument that it can be lifted by adding a small perturbation to the model. For instance, a weak repulsion between nearest neighbor sites has the desired effect. 
Let

$$
\psi=\sqrt{p} \psi^{(1,4)}+\sqrt{1-p} \psi^{(2,3)} .
$$

Projection of $\psi$ onto the eigenspace with eigenvalue -2 gives the former term, onto the eigenspace with eigenvalue +2 the latter term. The average linear entanglement is

$$
\overline{\mathcal{E}_{1}(\psi)}=\frac{1}{2}+p_{1,4} p_{2,3}=1 / 2+p(1-p) .
$$

\section{BOSONIC MODEL}

As an example of the bosonic case we consider a model which is similar to the boson-Hubbard model [32-34].

The bosonic creation and annihilation operators satisfy the commutation relations $\left[b_{j}, b_{k}^{\dagger}\right]=\delta_{j k}$. The Hamiltonian is given by

$$
H=+\sum_{j, k=1}^{N} t_{j k} b_{j}^{\dagger} b_{k} .
$$

However, unlike in the boson-Hubbard model, the hopping coefficients are not restricted to nearest neighbor. They rather satisfy

$$
t_{j k}=[1-(N-1) \epsilon] \delta_{j k}+\epsilon\left(1-\delta_{j k}\right) .
$$

This model is known as the Bose-Hubbard model with infinite range hopping [35].

Degeneracy is very important in this model. Indeed, assume $\epsilon>0$. Then the ground state of the one-particle Hamiltonian is $(N-1)$ fold degenerate. Hence the two-particle system has only three energy levels. We will consider the state $|1,1,0,0, \ldots, 0\rangle$, in which the bosons are not entangled. Next we calculate the average entanglement and show that it tends to $1 / 2$ when the size $N$ of the system becomes large.

\section{Projection onto invariant subspaces}

The one-particle ground state is $(N-1)$ fold degenerate with energy $1-N \epsilon$. Indeed, one calculates for $m \neq n$

$$
H\left(b_{m}^{\dagger}-b_{n}^{\dagger}\right)|0\rangle=\sum_{j}\left(t_{j m}-t_{j n}\right) b_{j}^{\dagger}|0\rangle=(1-N \epsilon)\left(b_{m}^{\dagger}-b_{n}^{\dagger}\right)|0\rangle .
$$

$N-1$ of these vectors $\left(b_{m}^{\dagger}-b_{n}^{\dagger}\right)|0\rangle$ are linearly independent. The remaining eigenstate, orthogonal to the ground states, has eigenvalue 1 . Its wave function is

$$
\frac{1}{\sqrt{N}} \sum_{j=1}^{N} b_{j}^{\dagger}|0\rangle=b^{\dagger}\left(\phi^{(0)}\right)|0\rangle,
$$

with

$$
\phi^{(0)}=\frac{1}{\sqrt{N}} \sum_{j} \omega_{j}
$$

and $\omega_{j}$ the one-particle basis formed by $\omega_{j}=b_{j}^{\dagger}|0\rangle$. Each of these basis vectors can be projected onto this eigenvector

$$
\omega_{j}=\frac{1}{\sqrt{N}} \phi^{(0)}+\xi_{j}
$$

The vectors $\xi_{j}$ are orthogonal to $\phi^{(0)}$ and hence belong to the degenerate space of eigenvectors.

The one-particle eigenfunction $\psi_{0}$ determines an eigenstate $\psi^{(00)}$ of the two-particle Hamiltonian by

$$
\psi^{(00)}=\frac{1}{\sqrt{2}} b^{\dagger}\left(\phi^{(0)}\right) b^{\dagger}\left(\phi^{(0)}\right)|0\rangle .
$$

The initial state

$$
\psi=|1,1,0,0, \ldots\rangle=b_{1}^{\dagger} b_{2}^{\dagger}|0\rangle
$$

is now projected onto the three invariant subspaces by writing it into the form

$$
\begin{aligned}
|1,1,0,0, \ldots\rangle= & \frac{1}{N} b^{\dagger}\left(\phi^{(0)}\right) b^{\dagger}\left(\phi^{(0)}\right)|0\rangle \\
& +\frac{1}{\sqrt{N}} b^{\dagger}\left(\phi^{(0)}\right) b^{\dagger}\left(\xi_{1}+\xi_{2}\right)|0\rangle+b^{\dagger}\left(\xi_{1}\right) b^{\dagger}\left(\xi_{2}\right)|0\rangle \\
\equiv & \sqrt{p^{(00)}} \psi^{(00)}+\sqrt{p^{(11)}} \psi^{(11)}+\sqrt{p^{(01)}} \psi^{(01)}, \quad \text { (56) }
\end{aligned}
$$

with normalized eigenfunctions $\psi^{(\sigma, \tau)}$ and normalization constants $p^{(\sigma, \tau)}$. It is straightforward to find that (see Appendix B)

$$
\begin{gathered}
p^{(00)}=\frac{2}{N^{2}}, \quad p^{(11)}=\left(1-\frac{1}{N}\right)^{2}+\frac{1}{N^{2}}, \\
p^{(01)}=\frac{2}{N}\left(1-\frac{2}{N}\right) .
\end{gathered}
$$

\section{Entanglement}

Next, one should decompose the eigenfunctions $\psi^{(00)}, \psi^{(11)}, \psi^{(01)}$ into the basis vectors

$$
\psi^{(\sigma, \tau)}=\sum_{j k} \lambda_{j k}^{(\sigma, \tau)} \omega_{j} \otimes \omega_{k} .
$$

The calculation of the matrices $\Lambda^{(00)}, \Lambda^{(11)}, \Lambda^{(01)}$ is found in Appendix B-see Eqs. (B11)-(B13). These are used to calculate the density matrices $\sigma \tau$, and the average entanglements

$$
\mathcal{E}(\sigma)=\mathcal{E}(\tau)=\frac{1}{2}+\frac{1}{N}-\frac{2}{N^{2}}
$$

and

$$
\Delta=\frac{1}{2}+\frac{2}{N}-\frac{8}{N^{2}}+\frac{16}{N^{3}}-\frac{16}{N^{4}} .
$$

See Appendix B. The final result is

$$
\overline{\mathcal{E}_{1}(\psi)}=\frac{1}{2}+\frac{4}{N^{2}}\left(1-\frac{2}{N}\right)^{2} \text {. }
$$

The average entanglement is always larger than $1 / 2$, is maximal at $N=4$ with a value of $9 / 16$, and converges as $1 / N^{2}$ towards $1 / 2$ for large $N$. 


\section{DISCUSSION}

In a rather long Introduction we have summed up a number of results that appeared in the literature. We have reviewed known properties of symmetric and antisymmetric matrices, with proofs in Appendix A. When applied to wave functions in a Fock space they lead to the definition of reduced density operators for systems consisting of two identical particles. These results are known. They generalize the Schmidt decomposition theorem to pairs of identical particles. We propose to take this generalized decomposition theorem as the basis for defining a measure of entanglement of two identical particles. Up to now, many authors have used for identical particles the same expressions as for distinguishable particles. This leads to the artificial result that the entanglement of two identical fermions is always larger than 1. Subtracting this constant 1 is not needed when using the definition (6).

In Sec. III, the technique to calculate the time-average entanglement is explained. The linear entropy is used instead of the von Neumann entropy in order to simplify the calculations. The extension of this technique to systems of two identical particles is straightforward, using the generalized Schmidt decomposition.

Two applications have been considered: One for fermions, the other for bosons. In the fermion model the average entanglement of two identical electrons can be calculated for arbitrary initial conditions. However, in this calculation, we have neglected the effect of degeneracy of the spectrum of the Hamiltonian. This can be justified with the argument that small perturbations would lift the degeneracy. The average entanglement obtained in this way is always larger than onehalf and is a nontrivial conserved quantity. In the boson model the degeneracy is much worse, leaving only three distinct energy levels. For one particular initial state we have shown that the average entanglement can be calculated, taking degeneracy into account. The resulting value tends to $1 / 2$ when the size of the system becomes large.

Related results have been obtained by other authors. Lévay et al. [26] consider two fermions in combination with a one-particle Hilbert space of dimension 4. Wang and Sanders [36] use the generalized decomposition theorem to decompose the state of the system into qubit states. Next they calculate the entanglement of one qubit with the others and average over the choice of qubits. Plenio et al. $[11,37,38]$ have considered the typical entanglement in ensembles of Gaussian states. These states differ considerably from the two-particle states considered here. Nevertheless, the matrix decomposition theorems might be relevant for their context as well.

Only bipartite entanglement has been considered in the present paper. Multipartite entanglement is more complicated and requires additional investigation. See for instance [39-41]. Neither did we study spatial entanglement of identical particles [42-44], or other measures of entanglement, like concurrence [44]. Finally, note that we assume that the time evolution is unitary. One expects that, due to interactions with the environment, entanglement will fade away. See the review paper [45].

\section{APPENDIX A}

For the sake of completeness, we give here a proof of theorems 1 and 2. First assume normal matrices.

Proposition 1. If $M$ is normal and symmetric then there exists an orthogonal matrix $V$ and a diagonal matrix $D$ such that $M=V^{T} D V$.

Proof. Let $\left\{E^{(n)}\right\}_{n}$ be a spectral family in a finite dimensional Hilbert space. Then there exists a unitary matrix $V$ and two-by-two disjunct sets $I_{n}$ such that

$$
E^{(n)}=V^{\dagger} \mathbb{I}^{(n)} V
$$

where

$$
\mathrm{I}_{p q}^{(n)}=1 \quad \text { if } p=q \in I_{n},=0 \quad \text { otherwise. }
$$

Note that

$$
E_{p q}^{(n)}=\sum_{r \in I_{n}} \overline{V_{r p}} V_{r q}
$$

Hence, if $E^{(n)}$ is symmetric then all elements $E_{p q}^{(n)}$ are real. This implies that, if all $E^{(n)}$ are symmetric, then $V$ can be chosen orthogonal, i.e., $V^{\dagger}=V^{T}$.

Let $M=\Sigma_{n} \lambda_{n} E^{(n)}$ be the spectral decomposition of $M$ with all $\lambda_{n}$ two by two distinct. Then also the $E^{(n)}$ are symmetric because of the uniqueness of the spectral decomposition and because the transpose of an orthogonal projection operator is again an orthogonal projection operator. Hence there exists an orthogonal matrix $V$ such that

$$
M=V^{T} D V \quad \text { with } D=\sum_{n} \lambda_{n} \mathbb{I}^{(n)} .
$$

If $M$ is antisymmetric then

$$
0=\sum_{n} \lambda_{n}\left[E^{(n)}+\left(E^{(n)}\right)^{T}\right] .
$$

This does not imply that the $E^{(n)}$ are antisymmetric (which is impossible for a nonvanishing orthogonal projection operator anyway) Hence a different line of reasoning is needed.

Proposition 2. If $M$ is normal and antisymmetric then there exists a unitary matrix $U$ such that $U^{T} M U$ has on each row and each column at most one nonvanishing element.

Proof. Let $M=\Sigma_{n} \lambda_{n} E^{(n)}$ be the spectral decomposition of $M$ with all $\lambda_{n}$ two by two distinct. Now assume $\zeta$ is an eigenvector of $M$ with eigenvalue $\lambda_{n} \neq 0$, satisfying $E^{(n)} \zeta$ $=\zeta$. Define $\eta$ by $\eta_{r}=\bar{\zeta}_{r}$. Then one has

$$
\begin{aligned}
(M \eta)_{r}=\sum_{s} M_{r s} \eta_{s} & =-\sum_{s} M_{s r} \bar{\zeta}_{s} \\
& =-\overline{\sum_{s}\left(M^{\dagger}\right)_{r s} \zeta_{s}}=-\overline{\left(M^{\dagger} \zeta\right)_{r}}=-\lambda_{n} \eta_{r} .
\end{aligned}
$$

Hence $\eta$ is an eigenvector of $M$ with eigenvalue $-\lambda_{n}$. This implies that either $\lambda_{n}=0$ or there exists $m \neq n$ such that $\lambda_{m}$ $=-\lambda_{n}$. In the latter case, $m$ and $n$ are matching indices and $E^{(m)}$ projects on all vectors $\eta$ obtained by taking element wise complex conjugation of all vectors in the range of $E^{(n)}$.

Now choose an orthonormal basis $\zeta^{(1)}, \zeta^{(2)}, \ldots, \zeta^{(q)}$ in the range of $E^{(n)}$ and a corresponding basis $\eta^{(1)}, \eta^{(2)}, \ldots, \eta^{(q)}$ in 
the range of $E^{(m)}$, with $\eta_{s}^{(j)}=\overline{\zeta_{s}^{(j)}}$. Do this for all nonvanishing pairs of eigenvalues $\lambda_{m}=-\lambda_{n}$. Complement this with an orthonormal basis in the nullspace of $M$, if present. Collect all these basis vectors as columns of a unitary matrix $U$. For a given $\zeta^{(j)}$ in the range of $E^{(n)}$ is, with some abuse of notation,

$$
\begin{aligned}
\left(U^{T} M U \delta_{j}\right)_{p} & =\left(U^{T} M U\right)_{r j} \\
& =\sum_{s}\left(U^{T} M\right)_{r s} \zeta_{s}^{(j)}=\lambda_{j} \sum_{s}\left(U^{T}\right)_{r s} \zeta_{s}^{(j)} \\
& =\lambda_{j} \sum_{s} \zeta_{s}^{(r)} \zeta_{s}^{(j)}=\lambda_{j}\left\langle\eta^{(r)} \mid \zeta^{(j)}\right\rangle .
\end{aligned}
$$

By construction, the latter vanishes for all but at most one value of $r$. This ends the proof.

Finally, the above results are generalized to arbitrary square matrices. The argument is that found in the proof of [2], lemma 1.

Let there be given a matrix $M$ which is either symmetric or antisymmetric. The matrix $M M^{\dagger}$ is Hermitian and can be diagonalized by means of a unitary matrix $U$, i.e., $U^{\dagger} M M^{\dagger} U$ is diagonal. Let $C=U^{\dagger} M\left(U^{\dagger}\right)^{T}$. Then $C$, like $M$, is either symmetric or antisymmetric. In addition it satisfies (using that $U^{\dagger} M M^{\dagger} U$ is diagonal and that $M^{T}= \pm M$ )

$$
C C^{\dagger}=U^{\dagger} M M^{\dagger} U=\left(U^{\dagger} M M^{\dagger} U\right)^{T}=U^{T} M^{\dagger} M\left(U^{T}\right)^{\dagger}=C^{\dagger} C .
$$

This means that $C$ is normal and that, by the previous propositions, there exists a unitary matrix $V$ such that $V^{T} C V$ has on each row and each column at most one nonvanishing element. The proof of the two theorems then follows easily.

\section{APPENDIX B}

Here we present the calculation of the time average entanglement of the initial boson state

$$
\psi=|1,1,0, \ldots, 0\rangle=b_{1}^{\dagger} b_{2}^{\dagger}|0\rangle .
$$

See Sec. IV.

The nondegenerate eigenvector of the two-particle Hamiltonian is

$$
\psi^{(00)}=\frac{1}{\sqrt{2}} b^{\dagger}\left(\phi^{(0)}\right) b^{\dagger}\left(\phi^{(0)}\right)|0\rangle=\frac{1}{N \sqrt{2}} \sum_{j, k} b_{j}^{\dagger} b_{k}^{\dagger}|0\rangle .
$$

It has eigenvalue 2 . The projection of $|1,1,0,0, \ldots\rangle$ onto this eigenvector is $\sqrt{p^{(00)}} \psi^{(00)}$ with $p^{(00)}=2 / N^{2}$.

Introduce vectors $\xi_{j}$, orthogonal to $\phi^{(0)}$, determined by

$$
\omega_{j}=\left\langle\phi^{(0)} \mid \omega_{j}\right\rangle \phi^{(0)}+\xi_{j}=\frac{1}{\sqrt{N}} \phi^{(0)}+\xi_{j} .
$$

Then one can write

$$
\begin{aligned}
|1,1,0,0, \ldots\rangle= & \frac{1}{N} b^{\dagger}\left(\phi^{(0)}\right) b^{\dagger}\left(\phi^{(0)}\right)|0\rangle+b^{\dagger}\left(\xi_{1}\right) b^{\dagger}\left(\xi_{2}\right)|0\rangle \\
& +\frac{1}{\sqrt{N}} b^{\dagger}\left(\phi^{(0)}\right) b^{\dagger}\left(\xi_{1}+\xi_{2}\right)|0\rangle .
\end{aligned}
$$

The projection of $|1,1,0,0, \ldots\rangle$ onto the $(N-1)^{2}$-fold degen- erate subspace equals $b^{\dagger}\left(\xi_{1}\right) b^{\dagger}\left(\xi_{2}\right)|0\rangle$. It is written as $\sqrt{p^{(11)}} \psi^{(11)}$ with

$$
\begin{aligned}
p^{(11)}=\| b^{\dagger}\left(\xi_{1}\right) b^{\dagger}\left(\xi_{2}\right)|0\rangle \|^{2} & =\left\langle\xi_{1} \mid \xi_{1}\right\rangle\left\langle\xi_{2} \mid \xi_{2}\right\rangle+\left|\left\langle\xi_{1} \mid \xi_{2}\right\rangle\right|^{2} \\
& =\left(1-\frac{1}{N}\right)^{2}+\frac{1}{N^{2}} .
\end{aligned}
$$

The projection of $|1,1,0,0, \ldots\rangle$ onto the remaining subspace equals

$$
\frac{1}{\sqrt{N}} b^{\dagger}\left(\phi^{(0)}\right) b^{\dagger}\left(\xi_{1}+\xi_{2}\right)|0\rangle .
$$

It is written as $\sqrt{p^{(01)}} \psi^{(01)}$ with

$p^{(01)}=\frac{1}{N} \| b^{\dagger}\left(\phi^{(0)}\right) b^{\dagger}\left(\xi_{1}+\xi_{2}\right)|0\rangle\left\|^{2}=\frac{1}{N}\right\| \xi_{1}+\xi_{2} \|^{2}=\frac{2}{N}\left(1-\frac{2}{N}\right)$.

Explicit expressions for the three eigenstates are

$$
\psi^{(00)}=\phi^{(0)} \otimes \phi^{(0)}=\frac{1}{N} \sum_{j k} \omega_{j} \otimes \omega_{k},
$$

$$
\begin{aligned}
\psi^{(11)}= & \frac{1}{\sqrt{p^{(11)}}} \frac{1}{\sqrt{2}}\left(\xi_{1} \otimes \xi_{2}+\xi_{2} \otimes \xi_{1}\right) \\
= & \frac{1}{\sqrt{p^{(11)}}} \frac{1}{\sqrt{2}}\left[\omega_{1} \otimes \omega_{2}+\omega_{2} \otimes \omega_{1}+\frac{2 N}{\phi^{(0)}} \otimes \phi^{(0)}\right. \\
& -\frac{1}{\sqrt{N}} \phi^{(0)} \otimes \omega_{1}-\frac{1}{\sqrt{N}} \omega_{1} \otimes \phi^{(0)}-\frac{1}{\sqrt{N}} \phi^{(0)} \\
& \left.\otimes \omega_{2}-\frac{1}{\sqrt{N}} \omega_{2} \otimes \phi^{(0)}\right],
\end{aligned}
$$

$$
\begin{aligned}
\psi^{(01)}= & \frac{1}{\sqrt{p^{(01)}}} \frac{1}{\sqrt{2 N}}\left[\phi^{(0)} \otimes\left(\xi_{1}+\xi_{2}\right)+\left(\xi_{1}+\xi_{2}\right) \otimes \phi^{(0)}\right] \\
= & \frac{1}{\sqrt{p^{(01)}}} \frac{1}{\sqrt{2 N}}\left[\phi^{(0)} \otimes\left(\omega_{1}+\omega_{2}\right)+\left(\omega_{1}+\omega_{2}\right) \otimes \phi^{(0)}\right. \\
& \left.-\frac{4}{\sqrt{N}} \phi^{(0)} \otimes \phi^{(0)}\right] .
\end{aligned}
$$

The coefficients of the expansion of each of the vectors $\psi^{(00)}, \psi^{(11)}$, and $\psi^{(01)}$ into the basis vectors $\omega_{j} \otimes \omega_{k}$ can be written as

$$
\lambda_{j k}^{(00)}=\frac{1}{2} x_{j k}^{(1)},
$$

$$
\begin{gathered}
\lambda_{j k}^{(11)}=\frac{1}{2 N \sqrt{2 p^{(11)}}}\left[2 x_{j k}^{(1)}-2 x_{j k}^{(3)}+N x_{j k}^{(2)}-N y_{j k}\right], \\
\lambda_{j k}^{(01)}=\frac{1}{N \sqrt{2 p^{(01)}}}\left[x_{j k}^{(3)}-2 x_{j k}^{(1)}\right],
\end{gathered}
$$

with 


$$
\begin{gathered}
x_{j k}^{(1)}=\frac{2}{N}, \\
x_{j k}^{(2)}=\left(\delta_{j 1}+\delta_{j 2}\right)\left(\delta_{k 1}+\delta_{k 2}\right), \\
x_{j k}^{(3)}=\delta_{j 1}+\delta_{k 1}+\delta_{j 2}+\delta_{k 2}, \\
y_{j k}=\left(\delta_{j 1}-\delta_{j 2}\right)\left(\delta_{k 1}-\delta_{k 2}\right) .
\end{gathered}
$$

The matrices $X^{(1)}, X^{(2)}, X^{(3)}$ span a simple Jordan algebra of the spin factor type (see [46], Sec. 2.9.7). The Jordan product is defined by

$$
A * B=\frac{1}{2}(A B+B A)
$$

One verifies that

$$
\begin{aligned}
& X^{(1)} * X^{(1)}=2 X^{(1)}, \\
& X^{(2)} * X^{(2)}=2 X^{(2)}, \\
& X^{(3)} * X^{(3)}=2 X^{(3)}+N X^{(2)}+N X^{(1)}, \\
& X^{(1) * X^{(2)}}=\frac{2}{N} X^{(3)}, \\
& X^{(1)} * X^{(3)}=2 X^{(1)}+X^{(3)}, \\
& X^{(2)} * X^{(3)}=X^{(3)}+2 X^{(2)}, \\
& Y * Y=2 Y, \\
& Y^{*} X^{(j)}=0, \quad j=1,2,3 .
\end{aligned}
$$

There exists a representation of the Jordan algebra with the above product rules in $R^{2}+R+R$, with the product rule

$$
(u, a, \lambda) *(v, b, \mu)=(a v+b u,\langle u \mid v\rangle+a b, \lambda \mu) . \quad(\mathrm{B} 27)
$$

Let $u^{(1)}$ and $u^{(2)}$ be two unit vectors satisfying $\left\langle u^{(1)} \mid u^{(2)}\right\rangle=$ $-1+4 / N$. Then one can identify

$$
\begin{gathered}
X^{(1)}=\left(u^{(1)}, 1,0\right), \\
X^{(2)}=\left(u^{(2)}, 1,0\right), \\
X^{(3)}=\frac{1}{2}\left(N\left(u^{(1)}+u^{(2)}\right), 4,0\right), \\
Y=(0,0,2) .
\end{gathered}
$$

With this representation is

$$
\sqrt{p^{(00)}} \Lambda^{(00)}=\frac{1}{N \sqrt{2}}\left(u^{(1)}, 1,0\right),
$$

$$
\begin{aligned}
\sqrt{p^{(11)}} \Lambda^{(11)} & =\frac{1}{2 N \sqrt{2}}\left[2 X^{(1)}-2 X^{(3)}+N X^{(2)}-N Y\right] \\
& =\frac{1}{2 N \sqrt{2}}\left(-(N-2) u^{(1)}, N-2,-2 N\right), \\
\sqrt{p^{(01)}} \Lambda^{(01)} & =\frac{1}{N \sqrt{2}}\left[X^{(3)}-2 X^{(1)}\right] \\
& =\frac{1}{2 N \sqrt{2}}\left((N-4) u^{(1)}+N u^{(2)}, 0,0\right) .
\end{aligned}
$$

It is now straightforward to calculate the squares

$$
p^{(00)}\left(\Lambda^{(00)}\right)^{2}=\frac{1}{N^{2}}\left(u^{(1)}, 1,0\right)
$$

$$
p^{(11)}\left(\Lambda^{(11)}\right)^{2}=\frac{1}{4 N^{2}}\left(-(N-2)^{2} u^{(1)},(N-2)^{2}, 2 N^{2}\right),
$$

$$
p^{(01)}\left(\Lambda^{(01)}\right)^{2}=\frac{1}{N^{2}}(0, N-2,0) .
$$

Summing these relations gives

$$
\sigma=\tau=\frac{1}{4 N}\left(-(N-4) u^{(1)}, N, 2 N\right) .
$$

Squaring again gives

$$
\sigma^{2}=\tau^{2}=\frac{1}{16 N^{2}}\left(-2 N(N-4) u^{(1)}, N^{2}+(N-4)^{2}, 4 N^{2}\right) .
$$

The trace of the matrix represented by $(u, a, \lambda)$ equals $2 a$ $+\lambda$. Hence one finds Eq. (59).

Let us finally calculate $\Delta$. Squaring again gives

$$
\begin{gathered}
{\left[p^{(00)}\left(\Lambda^{(00)}\right)^{2}\right]^{2}=\frac{2}{N^{4}}\left(u^{(1)}, 1,0\right),} \\
{\left[p^{(11)}\left(\Lambda^{(11)}\right)^{2}\right]^{2}=\frac{1}{8 N^{4}}\left(-(N-2)^{4} u^{(1)},(N-2)^{4}, 2 N^{4}\right),} \\
{\left[p^{(01)}\left(\Lambda^{(01)}\right)^{2}\right]^{2}=\frac{1}{N^{4}}\left(0,(N-2)^{2}, 0\right) .}
\end{gathered}
$$

This gives

$$
\begin{aligned}
\Delta= & 1-\operatorname{Tr}\left[p^{(00)}\left(\Lambda^{(00)}\right)^{2}\right]^{2}-\operatorname{Tr}\left[p^{(11)}\left(\Lambda^{(11)}\right)^{2}\right]^{2}-\operatorname{Tr}\left[p^{(01)}\right. \\
& \left.\times\left(\Lambda^{(01)}\right)^{2}\right]^{2}=\frac{1}{2}+\frac{2}{N}-\frac{8}{N^{2}}+\frac{16}{N^{3}}-\frac{16}{N^{4}}
\end{aligned}
$$


[1] M. A. Nielsen and I. L. Chuang, Quantum Computation and Quantum Information (Cambridge University Press, Cambridge, UK, 2000).

[2] J. Schliemann, J. I. Cirac, M. Kuś, M. Lewenstein, and D. Loss, Phys. Rev. A 64, 022303 (2001).

[3] G.-C. Ghirardi and L. Marinatto, Phys. Rev. A 70, 012109 (2004).

[4] G.-C. Ghirardi and L. Marinatto, Fortschr. Phys. 52, 1045 (2004).

[5] F. Buscemi, P. Bordone, and A. Bertoni, Phys. Rev. A 75, 032301 (2007).

[6] W. J. Munro, K. Nemoto, and A. G. White, J. Mod. Opt. 48, 1239 (2001).

[7] F. Verstraete and M. M. Wolf, Phys. Rev. Lett. 89, 170401 (2002)

[8] M. G. A. Paris, F. Illuminati, A. Serafini, and S. De Sienna, Phys. Rev. A 68, 012314 (2003).

[9] A. Olaya-Castro, N. F. Johnson, and L. Quiroga, J. Opt. B: Quantum Semiclassical Opt. 6, S 730 (2004).

[10] H. Li, X. G. Wang, and B. Hu, J. Phys. A 37, 10665 (2004).

[11] A. Serafini, M. G. A. Paris, F. Illuminati, and S. De Siena, J. Opt. B: Quantum Semiclassical Opt. 7, R19R36 (2005).

[12] F. Buscemi, P. Bordone, and A. Bertoni, Phys. Rev. A 73, 052312 (2006).

[13] X. W. Hou, M. F. Wan, and Z. Q. Ma, Chem. Phys. Lett. 426, 469 (2006).

[14] M. Lombardi and A. Matzkin, Phys. Rev. A 73, 062335 (2006).

[15] X. W. Hou and B. B. Hu, Phys. Rev. A 69, 042110 (2004).

[16] R. Demkowicz-Dobrzanski and M. Kuś, Phys. Rev. E 70, 066216 (2004).

[17] A. Lakshminarayan and V. Subrahmanyam, Phys. Rev. A 67, 052304 (2003).

[18] X. W. Hou, J. H. Chen, and B. Hu, Phys. Rev. A 71, 034302 (2005).

[19] Q. Xie and W. Hai, Eur. Phys. J. D 33, 265 (2005).

[20] R. Oliveira, O. C. O. Dahlsten, and M. B. Plenio, Phys. Rev. Lett. 98, 130502 (2007).

[21] O. C. O. Dahlsten, R. Oliveira, and M. B. Plenio, e-print arXiv:quant-ph/0701125.
[22] J. Naudts and E. Van der Straeten, J. Stat. Mech.: Theory Exp. 2006, P06015 (2006).

[23] T. Verhulst and J. Naudts, J. Phys. A 40, 2475 (2007).

[24] R. A. Horn and C. R. Johnson, Matrix Analysis (Cambridge University Press, Cambridge, UK, 1988).

[25] H. Eves, Elementary Matrix Theory (Dover, New York, 1980).

[26] P. Lévay, S. Nagy, and J. Pipek, Phys. Rev. A 72, 022302 (2005).

[27] B. Zumino, J. Math. Phys. 3, 1055 (1962).

[28] C. N. Yang, Rev. Mod. Phys. 34, 694 (1962).

[29] E. H. Lieb and F. Y. Wu, Phys. Rev. Lett. 20, 1445 (1968); 21, 192(E) (1968).

[30] E. H. Lieb and F. Y. Wu, Physica A 321, 1 (2003).

[31] T. Deguchi, F. H. L. Essler, F. Göhmann, A. Klümper, V. E. Korepin, and K. Kusakabe, Phys. Rep. 331, 197 (2000).

[32] M. P. A. Fisher, P. B. Weichman, G. Grinstein, and D. S. Fisher, Phys. Rev. B 40, 546 (1989).

[33] D. Ueltschi, Prog. Probab. 51, 363 (2002).

[34] O. Romero-Isart, K. Eckert, C. Rodó, and A. Sanpera, e-print arXiv:quant-ph/0703177.

[35] J.-B. Bru and T. C. Dorlas, J. Stat. Phys. 113, 177 (2003).

[36] X.-G. Wang and B. C. Sanders, J. Phys. A 38, L67 (2005).

[37] A. Serafini, O. C. O. Dahlsten, and M. B. Plenio, e-print arXiv:quant-ph/0610090.

[38] A. Serafini, O. C. O. Dahlsten, D. Gross, and M. B. Plenio, e-print arXiv:quant-ph/0701051.

[39] L. Chen and Y.-X. Chen, Phys. Rev. A 74, 062310 (2006).

[40] N. Kiesel, C. Schmid, G. Tóth, E. Solano, and H. Weinfurter, Phys. Rev. Lett. 98, 063604 (2007).

[41] T. Vertési, e-print arXiv:quant-ph/0701246.

[42] L. Heaney, J. Anders, and V. Vedral, e-print arXiv:quant-ph/ 0607069.

[43] M. T. L. Hsu, W. P. Bowen, N. Treps, and P. K. Lam, Phys. Rev. A 72, 013802 (2005).

[44] A. Ramsak, I. Sega, and J. H. Jefferson, Phys. Rev. A 74, 010304(R) (2006).

[45] F. Mintert, A. R. R. Carvalho, M. Kuś, and A. Buchleitner, Phys. Rep. 415, 207 (2005).

[46] H. Hanche-Olsen and E. Størmer, Jordan Operator Algebras (Pitman Advanced Publishing Program, Boston, MA, 1984). 